\section{UJMM

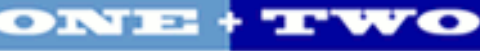

Volume 7| 2017 Spring 2017

\section{Undergraduate Journal of Mathematical} Modeling: One + Two

2017

\title{
Diagnosing Breast Cancer with a Neural Network
}

John Cullen

University of South Florida

\author{
Advisors: \\ Arcadii Grinshpan, Mathematics and Statistics \\ John Cullen Sr., Principal Software Engineer, Mach7 Technologies \\ Problem Suggested By: John Cullen Sr.
}

Follow this and additional works at: https://digitalcommons.usf.edu/ujmm

Part of the Mathematics Commons

UJMM is an open access journal, free to authors and readers, and relies on your support: Donate Now

\section{Recommended Citation}

Cullen, John (2017) "Diagnosing Breast Cancer with a Neural Network," Undergraduate Journal of Mathematical Modeling: One + Two: Vol. 7: Iss. 2, Article 4.

DOI: http://doi.org/10.5038/2326-3652.7.2.4880

Available at: https://digitalcommons.usf.edu/ujmm/vol7/iss2/4 


\title{
Diagnosing Breast Cancer with a Neural Network
}

\begin{abstract}
Fine needle aspiration (FNA) is a minimally invasive biopsy technique that can be used to successfully diagnose types of cancer, including breast cancer. Immediately, it is difficult for a human to spot any trends in the cell level data gathered during a fine needle aspiration procedure. One way to predict the type of tumor a patient has, is to use a computer to develop a mathematical model based on known data. This project utilizes the Diagnostic Wisconsin Breast Cancer Database (DWBCDB) to create an accurate mathematical model that predicts the type of a patient's tumor (Malignant or Benign). A neural network model is created in a two step-process. It is first created with random parameters, and is then refined using the data set, with known tumor types. A model with a success rate of $98 \%$ is created, which suggests that there is a high level of correlation between FNA data and the type of tumor a patient had. This approach was not capable of producing a perfect model that could be used in clinical applications.
\end{abstract}

\section{Keywords}

breast cancer, machine learning, neural network

\section{Creative Commons License}

(c)

This work is licensed under a Creative Commons Attribution-Noncommercial-Share Alike 4.0 License. 


\section{PROBLEM STATEMENT}

Using a large set of Fine Needle Aspirate (FNA) breast cancer biopsy data, develop a model that can accurately predict whether a new patient's tumor is malignant or benign.

\section{Motivation}

The issue of finding noninvasive techniques for the diagnosis of diseases is extremely important. Generally, surgical biopsies can be very anxiety provoking in patients, and may even lead them to put off the procedure or not get it done at all. This of course, will reduce their chance of survival if they do have the disease. Techniques like FNA are less threatening to patients, safer, and faster. Techniques like this often do not provide as much information to a doctor as a full biopsy would. In this difficult situation, a computer can be used to recognize and diagnose patients based on a mathematical model that it has developed based on previous patients with known tumor types.

Given a large data set, we know some basic common sense information about the trends it contains, but we don't have a way to quantify this, or the complex interactions each variable has with one another. The field of machine learning allows computers to quantify these interactions for us, and is very important to the computer science field. There are applications for machine learning in the medical field, artificial intelligence, marketing, speech recognition, and many other areas of study. Neural networks can be used successfully on a diverse set of problems, and have started to become very powerful as computer hardware improves. Models that are trained by a computer, or more specifically, neural networks, can pick up very difficult to spot trends that humans often miss. This allows us to develop very accurate prediction systems without needing to exhaustively analyze data. 
The objective of this project is to develop an accurate neural network based model with machine learning techniques, that is capable classifying a patient's tumor type based on the data found in the DWBCDB.

\section{MATHEMATICAL DESCRIPTION AND SOLUTION APPROACH}

\section{MODELING THE DATA}

Table 1 covers each data point in the DWBCDB, as well as the known type of the patient's tumor. We will be using this data set to develop a mathematical model to predict a new patient's tumor type.

\begin{tabular}{|c|c|}
\hline Variables & Description \\
\hline $\begin{array}{l}\text { 1. Mean (Fields 1-10) } \\
\text { a. Radius } \\
\text { b. Texture } \\
\text { c. Perimeter } \\
\text { d. Area } \\
\text { e. Smoothness } \\
\text { f. Compactness } \\
\text { g. Concavity } \\
\text { h. Concave Points } \\
\text { i. Symmetry } \\
\text { j. } \text { Fractal Dimension }\end{array}$ & $\begin{array}{l}\text { The means of the calculated cell values from the } \\
\text { sample. }\end{array}$ \\
\hline $\begin{array}{l}\text { 2. Standard error (Fields 11-20) } \\
\text { a. Radius } \\
\text { b. Texture } \\
\text { c. Perimeter } \\
\text { d. Area } \\
\text { e. Smoothness } \\
\text { f. Compactness } \\
\text { g. Concavity } \\
\text { h. Concave Points } \\
\text { i. Symmetry } \\
\text { j. } \\
\text { Fractal Dimension }\end{array}$ & $\begin{array}{l}\text { The standard errors of the calculated cell values } \\
\text { from the sample. }\end{array}$ \\
\hline $\begin{array}{l}\text { 3. Largest (Fields 21-30) } \\
\text { a. Radius } \\
\text { b. Texture } \\
\text { c. Perimeter }\end{array}$ & $\begin{array}{l}\text { The averages of the three largest values in the } \\
\text { sample of the values calculated for each cell. }\end{array}$ \\
\hline
\end{tabular}




\begin{tabular}{|l|l|}
\hline d. Area & \\
e. Smoothness & \\
f. Compactness & \\
g. Concavity & \\
h. Concave Points & \\
i. Symmetry & \\
j. $\quad$ Fractal Dimension & The type of the tumor that has developed in the \\
\hline 4. Diagnosis (Field 31) & patient's breast. M (Malignant) or B (Benign) \\
\hline
\end{tabular}

Table 2: A table of the parameters provided in the DWBCDB.

In a situation with many possibly correlated variables, one of the best options is to use a neural network to model the data and to make a prediction. A neural network is simply a mathematical model that can fit many shapes, unlike a low degree polynomial. It is based on the neuron structure of the human brain, but it much less powerful. A neural network can be 'taught' to recognize trends by adjusting its parameters so that the difference between what it predicts and the actual value is minimal.

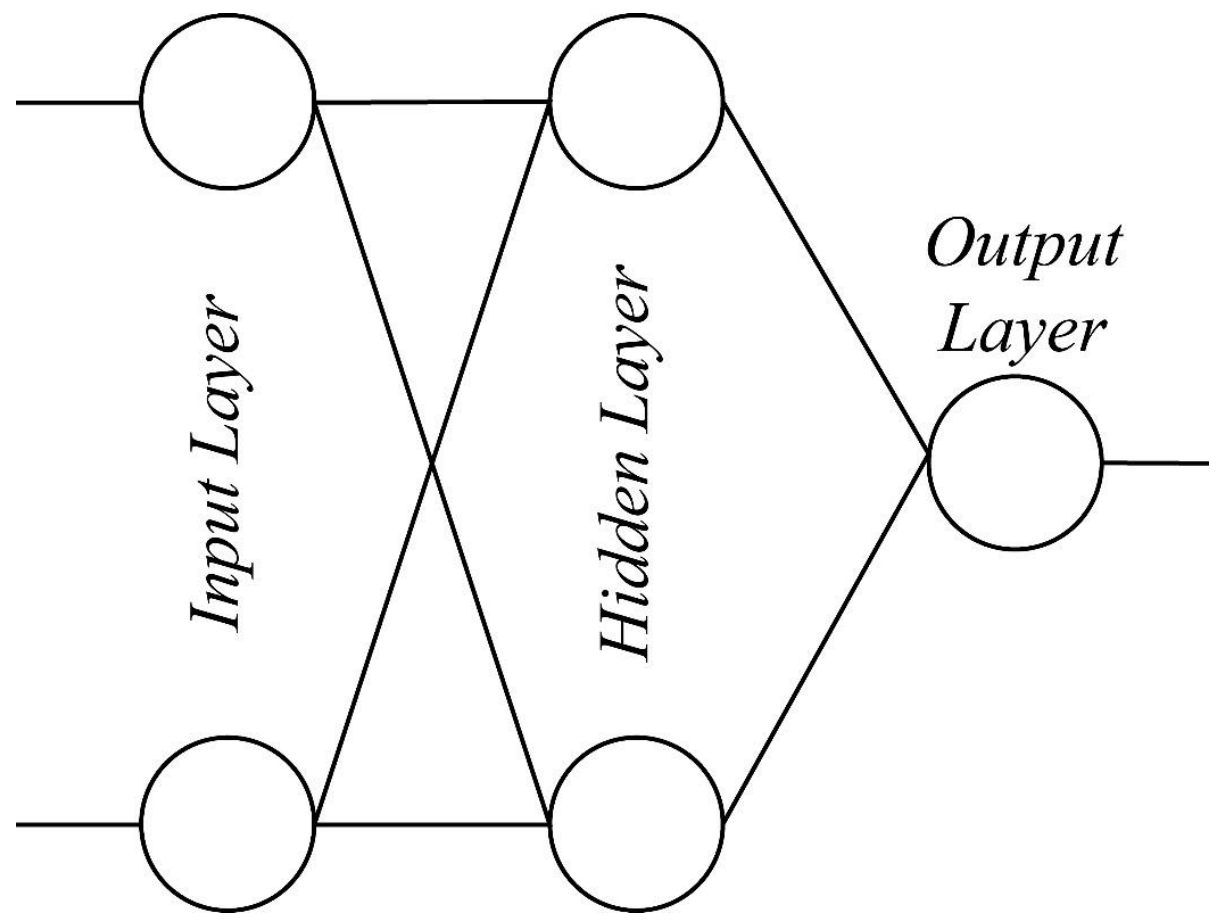

Figure 1: A simple neural Network. 
Figure 1 is a visual representation of a simple neural network. Each line represents a weight and each circle or node represents the weighted sum of the outputs of the previous layer that is run through a non-linear function called an activation function. The last layer, or the output layer, represents what the model has predicted based on the values entered in the first layer, or input layer. Although it is possible to have many output nodes, this situation will only require one. We will consider an output value of 1 to represent 'Malignant' and a value of 0 to represent 'Benign'. We can consider the network to be a multivariate function with a range of $(0,1)$.

$$
P=f_{\text {network }}\left(I_{1}, I_{2}, I_{3}, \ldots\right)
$$

When each node in the input layer is set to an activation value representing the input of the function, each subsequent node in the network will receive an activation value based on the ones in the previous layer, and the weights. The activation values in the first layer are simply the inputs of the network function, shown above. The activation value of the node in the last layer will be the prediction of the network. The activation for the $\mathrm{j}^{\text {th }}$ node in the $\ell^{\text {th }}$ layer is denoted by:

$$
a_{j}^{\ell}=\sigma\left(z_{j}^{\ell}\right)
$$

$\sigma(x)$ is a non-linear function. The function must be non-linear for the network to fit nonlinear data. We will use the sigmoid function because it closely resembles how a human neuron would be activated (Wikipedia).

$$
\begin{gathered}
\sigma(x)=\frac{1}{1+e^{-x}} \\
\sigma^{\prime}(x)=\sigma(x)(1-\sigma(x)) \\
\lim _{x \rightarrow \infty} \sigma(x)=1 \\
\lim _{x \rightarrow-\infty} \sigma(x)=0
\end{gathered}
$$




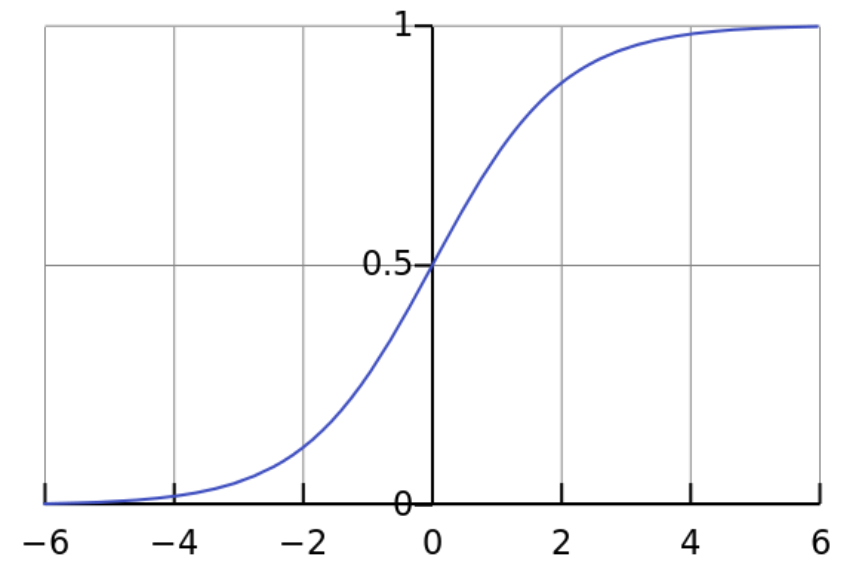

Figure 2: The logistic curve, $\sigma(x)$ (Wikipedia)

The limits of the sigmoid function are useful because we can consider an output close to 1 to represent an activated neuron and an output close to 0 to represent a non-activated neuron. They also scale all outputs to our desired range of $(0,1)$. In the output layer an activated neuron will mean that the data satisfies the criteria of the category that the node represents.

The term $z_{j}^{\ell}$ is the weighted sum of the inputs plus a bias term $b_{j}^{\ell}$. The bias constant is necessary here because we want the sigmoid function to be able to shift to the left or right, which makes fitting the function to the data easier. Later, both the weights and biases will be updated to make the network fit the data more closely, but they will be initialized to random constants.

$$
z_{j}^{\ell}=\sum_{i}^{n_{\ell-1}} \omega_{i j}^{\ell} a_{i}^{\ell-1}+b_{j}^{\ell}
$$

Here we are summing over each node $\mathrm{i}$ in the previous layer and multiplying its weight by its activation value. The upper limit of the sum, $n_{\ell-1}$, is the number of nodes in the previous layer. The term $\omega_{i j}^{\ell}$ represents the weight from the $\mathrm{i}^{\text {th }}$ node in the previous layer $(\ell-1)$ to the $\mathrm{j}^{\text {th }}$ node in the layer $\ell . a_{i}^{\ell-1}$ is the activation value of the $\mathrm{i}^{\text {th }}$ node in the previous layer. With equations (1) and (2), we can calculate the output of the neural network given the inputs. A completed application of these equations is in Appendix I. 


\section{Training THE Model: AdJUSting THE Weights AND BiAses}

To improve the weights and biases of the network, we can come up with a function of these parameters that returns a value that represents how far off the real value the network's prediction is. The parameters of the function can then be altered in a way that minimizes it. We will use the one half squared error function which is commonplace in machine learning applications. $y_{i}$ is the expected activation value for node $\mathrm{i}$ in the last layer, L. Note that in this equation the activations of the final layer are constants, as are the expected activations, because we will iterate through each training example and minimize the error for each example.

$$
E\left(\omega_{1,1}^{1}, \ldots \omega_{i j}^{\ell}, b_{1}^{1}, \ldots b_{j}^{\ell}\right)=\frac{1}{2} \sum_{i}^{n_{\mathrm{L}}}\left(y_{i}-a_{i}^{\mathrm{L}}\right)^{2}
$$

While it is theoretically possible to minimize the function in closed form, it would be very difficult and time consuming. Since we will be using a computer to do the math, we can use an iterative algorithm called gradient descent. Gradient descent uses the derivative of a function to find a downward slope, and then takes a step down that slope, much like the way a sled would go down a hill. The derivative is multiplied by a constant $\eta$, to determine how large the step should be. Note that this technique will only find a local minimum, so the process is repeated multiple times starting at random values, and the best result (Closest to absolute minimum) is used. There is an example of this process in Appendix II. Here is the equation that will be used to update each weight and bias using gradient descent:

$$
\begin{gathered}
\omega_{i j}^{\ell}:=\omega_{i j}^{\ell}-\eta \cdot \frac{\partial E}{\partial \omega_{i j}^{\ell}} \\
b_{j}^{\ell}:=b_{j}^{\ell}-\eta \cdot \frac{\partial E}{\partial b_{j}^{\ell}}
\end{gathered}
$$


Now all that is needed is the partial derivatives of $\mathrm{E}$ with respect to each weight and bias. These could be calculated using only the chain rule, but the process would be lengthy. A simple algorithm called Backpropagation will make the process faster. Backpropagations states (Nielsen):

$$
\delta_{j}^{\ell}=\frac{\partial E}{\partial z_{j}^{\ell}}
$$

This alias for the partial derivative of $\mathrm{E}$ with respect to $z_{j}^{\ell}$ is called the error or delta term, and can be used to calculate the error terms in the layer before it. To incorporate (6) into equations (4) and (5), the chain rule is applied:

$$
\begin{gathered}
\frac{\partial E}{\partial \omega_{i j}^{\ell}}=\frac{\partial E}{\partial z_{j}^{\ell}} \cdot \frac{\partial z_{j}^{\ell}}{\partial \omega_{i j}^{\ell}}=\delta_{j}^{\ell} \frac{\partial z_{j}^{\ell}}{\partial \omega_{i j}^{\ell}} \\
\frac{\partial E}{\partial b_{j}^{\ell}}=\frac{\partial E}{\partial z_{j}^{\ell}} \cdot \frac{\partial z_{j}^{\ell}}{\partial b_{j}^{\ell}}=\delta_{j}^{\ell} \frac{\partial z_{j}^{\ell}}{\partial b_{j}^{\ell}}
\end{gathered}
$$

The final term of each equation is easy to calculate, we take the partial derivatives of (2) with respect to the weights and biases. The summation may be removed because the term will be zero unless $\mathrm{k}$ is equal to $\mathrm{i}$ :

$$
\begin{gathered}
\frac{\partial z_{j}^{\ell}}{\partial \omega_{i j}^{\ell}}=\frac{\partial}{\partial \omega_{i j}^{\ell}}\left(\sum_{k}^{n_{\ell-1}} \omega_{k j}^{\ell} a_{k}^{\ell-1}+b_{j}^{\ell}\right)=\frac{\partial}{\partial \omega_{i j}^{\ell}}\left(\omega_{i j}^{\ell} a_{i}^{\ell-1}+b_{j}^{\ell}\right)=a_{i}^{\ell-1} \\
\frac{\partial z_{j}^{\ell}}{\partial b_{j}^{\ell}}=\frac{\partial}{\partial b_{j}^{\ell}}\left(\sum_{k}^{n_{\ell-1}} \omega_{k j}^{\ell} a_{k}^{\ell-1}+b_{j}^{\ell}\right)=1
\end{gathered}
$$

The partial derivatives of the error function, (3), may be revised to:

$$
\frac{\partial E}{\partial \omega_{i j}^{\ell}}=\delta_{j}^{\ell} \frac{\partial z_{j}^{\ell}}{\partial \omega_{i j}^{\ell}}=\delta_{j}^{\ell} \cdot a_{i}^{\ell-1}
$$




$$
\frac{\partial E}{\partial b_{j}^{\ell}}=\delta_{j}^{\ell} \frac{\partial z_{j}^{\ell}}{\partial b_{j}^{\ell}}=\delta_{j}^{\ell}
$$

To find delta in terms of the delta in the layer $\ell+1$, the chain rule can be applied to (6) to find how the $\mathrm{z}$ value of one node affects all the $\mathrm{z}$ values in the next layer, and subsequently how those $\mathrm{z}$ values affect the one-half squared error function.

$$
\delta_{j}^{\ell}=\frac{\partial E}{\partial z_{j}^{\ell}}=\sum_{k}^{n_{\ell+1}} \frac{\partial E}{\partial z_{k}^{\ell+1}} \cdot \frac{\partial z_{k}^{\ell+1}}{\partial z_{j}^{\ell}}=\sum_{k}^{n_{\ell+1}} \delta_{k}^{\ell+1} \cdot \frac{\partial z_{k}^{\ell+1}}{\partial z_{j}^{\ell}}
$$

The summation of the values for each node in the next layer is required because the change of a node in a previous layer will affect all nodes in the next layer, and all layers after that. The delta alias can be substituted for the first partial derivative, representing the error of each node in the next layer. The partial derivative of $z_{k}^{\ell+1}$ can be calculated like so, using a slightly modified version of (2):

$$
\frac{\partial z_{k}^{\ell+1}}{\partial z_{j}^{\ell}}=\frac{\partial}{\partial z_{j}^{\ell}}\left(\sum_{i}^{n_{\ell}} \omega_{i k}^{\ell+1} a_{i}^{\ell}+b_{k}^{\ell+1}\right)
$$

The summation can be removed because all terms where $i \neq j$ will be zero. We also substitute $\sigma\left(z_{i}^{\ell}\right)$ in for $a_{i}^{\ell}$ using (1).

$$
\frac{\partial z_{k}^{\ell+1}}{\partial z_{j}^{\ell}}=\frac{\partial}{\partial z_{j}^{\ell}}\left(\omega_{j k}^{\ell+1} \sigma\left(z_{j}^{\ell}\right)+b_{k}^{\ell+1}\right)=\omega_{j k}^{\ell+1} \sigma^{\prime}\left(z_{j}^{\ell}\right)
$$

Based on the above equations, we can use the following equation for the delta of an arbitrary node $\mathrm{j}$ in layer $\ell$, where $\mathrm{k}$ represents a node in the next layer, $\ell+1$ :

$$
\delta_{j}^{\ell}=\sum_{k}^{n_{\ell+1}} \delta_{k}^{\ell+1} \cdot \omega_{j k}^{\ell+1} \sigma^{\prime}\left(z_{j}^{\ell}\right)
$$


Now we just need to determine the actual value of $\delta_{j}^{\ell}$ in the last layer, so we can use it to solve the rest of the delta terms. This can be solved simply by substituting $\sigma\left(z_{i}^{\mathrm{L}}\right)$ in for $a_{i}^{\mathrm{L}}$ using (1), and applying the chain rule. The summation can be eliminated again because if $\mathrm{i} \neq \mathrm{j}$ the derivative will be zero.

$$
\begin{gathered}
\delta_{j}^{\mathrm{L}}=\frac{\partial E}{\partial z_{j}^{\mathrm{L}}}=\frac{\partial}{\partial z_{j}^{\mathrm{L}}}\left(\frac{1}{2} \sum_{i}^{n_{\mathrm{L}}}\left(y_{i}-\sigma\left(z_{i}^{\mathrm{L}}\right)\right)^{2}\right)=\frac{\partial}{\partial z_{j}^{\mathrm{L}}}\left(\frac{1}{2}\left(y_{j}-\sigma\left(z_{j}^{\mathrm{L}}\right)\right)^{2}\right) \\
=\left(y_{j}-\sigma\left(z_{j}^{\mathrm{L}}\right)\right)\left(0-\sigma^{\prime}\left(z_{j}^{\mathrm{L}}\right)\right) \\
=-\left(y_{j}-a_{j}^{\mathrm{L}}\right) \sigma^{\prime}\left(z_{j}^{\mathrm{L}}\right) \\
=\left(a_{j}^{\mathrm{L}}-y_{j}\right) \sigma^{\prime}\left(z_{j}^{\mathrm{L}}\right)
\end{gathered}
$$

The last layer's delta term can be used to calculate all other error terms, and then the partial derivatives of each weight and bias, which gives us enough information to complete an update of the parameters to minimize the error function. A worked example of a weight update can be found in Appendix III.

\section{IMPLEMENTING THE MODEL}

Fully training the model to fit the dataset will require thousands of iterations of gradient descent with hundreds of training examples. For this reason, the most efficient way to implement the model is by creating a computer program that will make the calculations without the possibility of human error. The following is an explanation of the program that I implemented, the full source code can be found in Appendix IV.

1. Before writing the program, I scaled all the data from the DWBCDB into values between 0 and 1 . This makes the input activation levels fall in the same range as the rest of the 
activation values. This was done using the following formula, where $\mathrm{x}$ is the column's value for a row of data: $\frac{x-x_{\min }}{x_{\max }-x_{\min }}$.

2. Separate the data set into two distinct sets, one to use for training, and the other to test the model as it is trained. The DWBCDB has 569 different patients in it. I chose a training set of 469 rows, so that the testing set would be 100 rows. This way the test results can easily be converted to a percentage.

3. A neural network with 3 layers is created. The first layer has 30 nodes, the number of the inputs. The second layer has 30 more nodes, and the output has one node where any value over 0.5 is 'Malignant' and any value under that threshold is 'Benign'. All the weights and biases are initialized to random values. Anything more than this number of layers makes the process extremely slow due to the increase in biases and weights that need to be updated.

4. For each row in the training set, an update of each bias and weight is performed, using the gradient descent equations. I found through trial and error that $\eta=0.02$ was a good value for the constant in the gradient descent equation. It takes steps big enough that the model learns quickly, but does not overshoot.

5. The model is tested using the 100-row training set. The network is fed the inputs for those rows, and the value of the output node is compared to the real output. The number correct guesses, as well as the mean of the error function (calculated for each row) is logged.

6. Steps 4-5 are repeated 1200 times. I found that this number provides the best results. If the process is run more than this, the model becomes too specific to the training set and begins to start failing to recognize items in the testing set. 
7. The entire process is repeated multiple times and the best result is used, in case the gradient descent algorithm finds a local minimum and gets stuck.

\section{DISCUSSION}

Running the program multiple times, I found a set of weights and biases that could predict the type of tumor in 98 of the 100 testing rows. This gives the model a $98 \%$ success rate. The exhaustive list of optimal weights and biases can be found in Appendix V. This is a higher success rate than anticipated and is indicative that at least some of the input parameters used have a very strong correlation with the output. Unfortunately, the model abstracts away a lot of information about how things are correlated so it is very difficult for a human to understand exactly what the machine is doing. It does however, provide a way to classify information when humans do not know anything about how it correlates to the results.

It is also clear in

Figure 3 that overfitting

was a problem that was

encountered during the

training process. The error

function for the 100

testing rows started to

increase even as the

machine should be

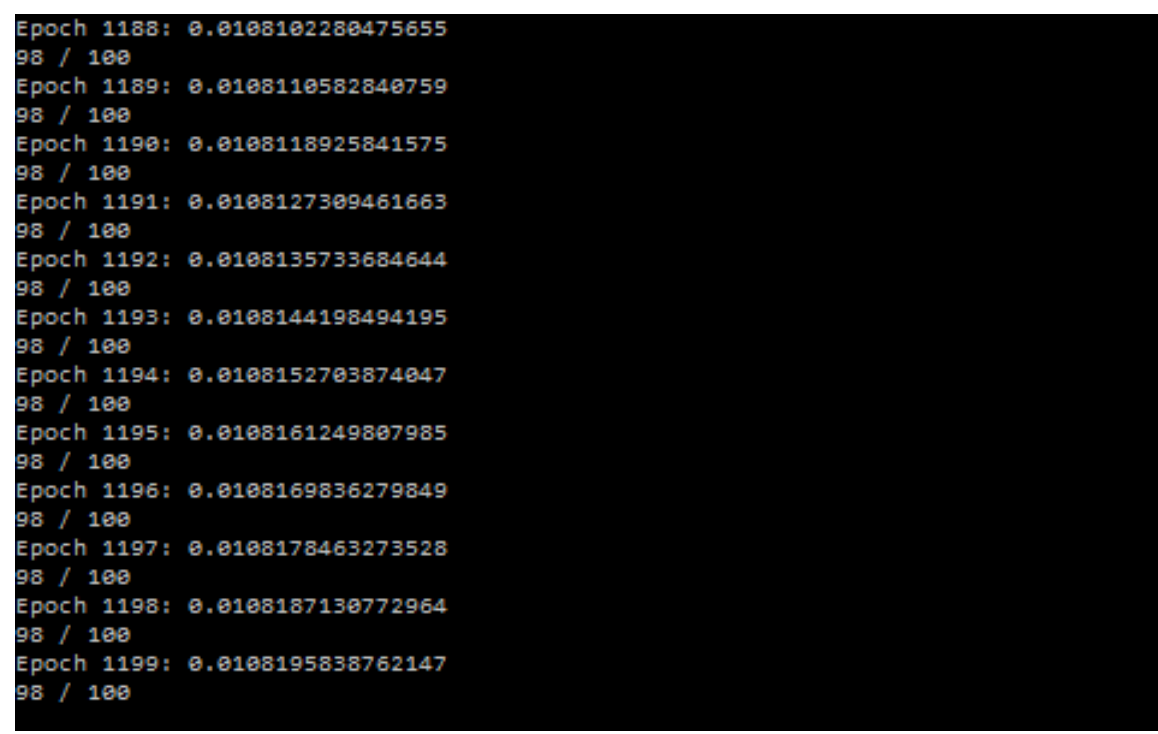

Figure 3: The output of the neural network program, found in Appendix IV.

perfecting its model. This means that the model was starting to specifically fit the training dataset 
and becoming less generic, thus making it unable to deal with items it has not seen before. This indicates the training set may need to be larger than it is, with a more diverse set of examples.

\section{CONCLUSION AND RECOMMENDATIONS}

Using a neural network system, a 98\% accurate model was reached. This suggests that there is a strong correlation between FNA data and the type of a patient's tumor. This model however was not $100 \%$ accurate which can pose some serious problems when diagnosing patients with cancer. The consequences of a false positive or a false negative are both potentially catastrophic to the patients' health. This model should not be used in a clinical setting. The model is also very abstract and doesn't allow humans to see what trends the computer is seeing.

Improvements in accuracy would be necessary to use this model in a clinical environment. Several techniques could improve the accuracy of the model. A wide array of activation functions could be tested and the best selected. A gradient descent technique that incorporates momentum, much like the real world, could help the model to reach a global minimum. A larger training data set could provide the system with more possible cases. Finally, other machine learning models could be considered. Some other models, like decision trees, or grouping allow humans to better interpret trends. 


\section{NOMENCLATURE}

\begin{tabular}{cl}
\hline Symbol & \multicolumn{1}{c}{ Description } \\
\hline \hline$a_{j}^{\ell}$ & The activation value of the node $\mathrm{j}$ in the layer $\ell$ \\
$z_{j}^{\ell}$ & $\begin{array}{l}\text { The weighted sum of the activations of the previous layer, plus the bias } \\
\text { term for node } \mathrm{j} \text { in layer } \ell\end{array}$ \\
$n_{\ell}$ & The number of nodes in the layer $\ell$ \\
$\sigma(x)$ & The sigmoid activation function \\
$\omega_{i j}^{\ell}$ & The weight between the node $\mathrm{i}$ in layer $\ell-1$ and the node $\mathrm{j}$ in $\ell$ \\
$b_{j}^{\ell}$ & The bias term for node $\mathrm{j}$ in layer $\ell$ \\
$E$ & The error function \\
$y_{i}$ & The expected output of node $\mathrm{i}$ in the output layer, based on the training set \\
$L$ & The last layer \\
$\eta$ & The 'learning rate' constant for the gradient descent equation \\
$\delta_{j}^{\ell}$ & The backpropagation error term. Same as $\frac{\partial E}{\partial z_{j}^{\ell}}$ \\
$:=$ & The assignment operator. Sets a variable to the specified value.
\end{tabular}

\section{REFERENCES}

Johnson, Justin and Andrej Karpathy. "CS231n: Convolutional Neural Networks for Visual Recognition." 2017. Github. Course Notes. May 2017. 〈http://cs231n.github.io/>.

Lichman, M. UCI Machine Learning Repository. 2013. University of California, School of Information and Computer Science. Database. May 2017. $<$ http://archive.ics.uci.edu/ml/>.

Mazur, Matt. "A Step by Step Backpropagation Example." 17 March 2015. Matt Mazur. Blog Post. May 2017. <https://mattmazur.com/2015/03/17/a-step-by-step-backpropagationexample/>. 
Neural Networks Demystified [Part 3: Gradient Descent]. Welch Labs. 2014. Short Video. $<$ https://www.youtube.com/watch?v=5u0jaA3qAGk>.

Neural Networks Demystified [Part 4: Backpropagation]. Welch Labs. 2014. Short Video. <https://www.youtube.com/watch?v=GlcnxUlrtek\&t=2s>.

Neural Networks Demystified [Part 5: Numerical Gradient Checking]. Welch Labs. 2014. Short Video. <https://www.youtube.com/watch?v=pHMzNW8Agq4>.

Nielsen, Michael. Neural Networks and Deep Learning. Michael Nielsen, 2017. E-Book.

Rashid, Tariq. Make Your Own Neural Network. CreateSpace Independant Publishing, 2016. PDF.

Ruder, Sebastian. "An overview of gradient descent optimization algorithms." 19 January 2016. Blog Post. May 2017. <http://sebastianruder.com/optimizing-gradient-descent/>. Shiffman, Daniel. The Nature of Code: Simulating Natural Systems with Processing. Daniel Shiffman, 2012. E-Book.

Wikipedia. "Sigmoid function." 1 May 2017. Wikipedia. Wiki. May 2017. <https://en.wikipedia.org/wiki/Sigmoid_function>.

Wolberg, Dr. William H., W. Nick Street and Olvi L. Mangasarian. "Breast Cancer Wisconsin (Diagnostic) Data Set." UCI Machine Learning Repository. UCI Machine Learning Repository, 0111 1995. CSV. <http://archive.ics.uci.edu/ml/datasets/Breast+Cancer+Wisconsin+(Diagnostic) >. 


\section{APPENDIX}

\section{FORWARD PROPAGATION}

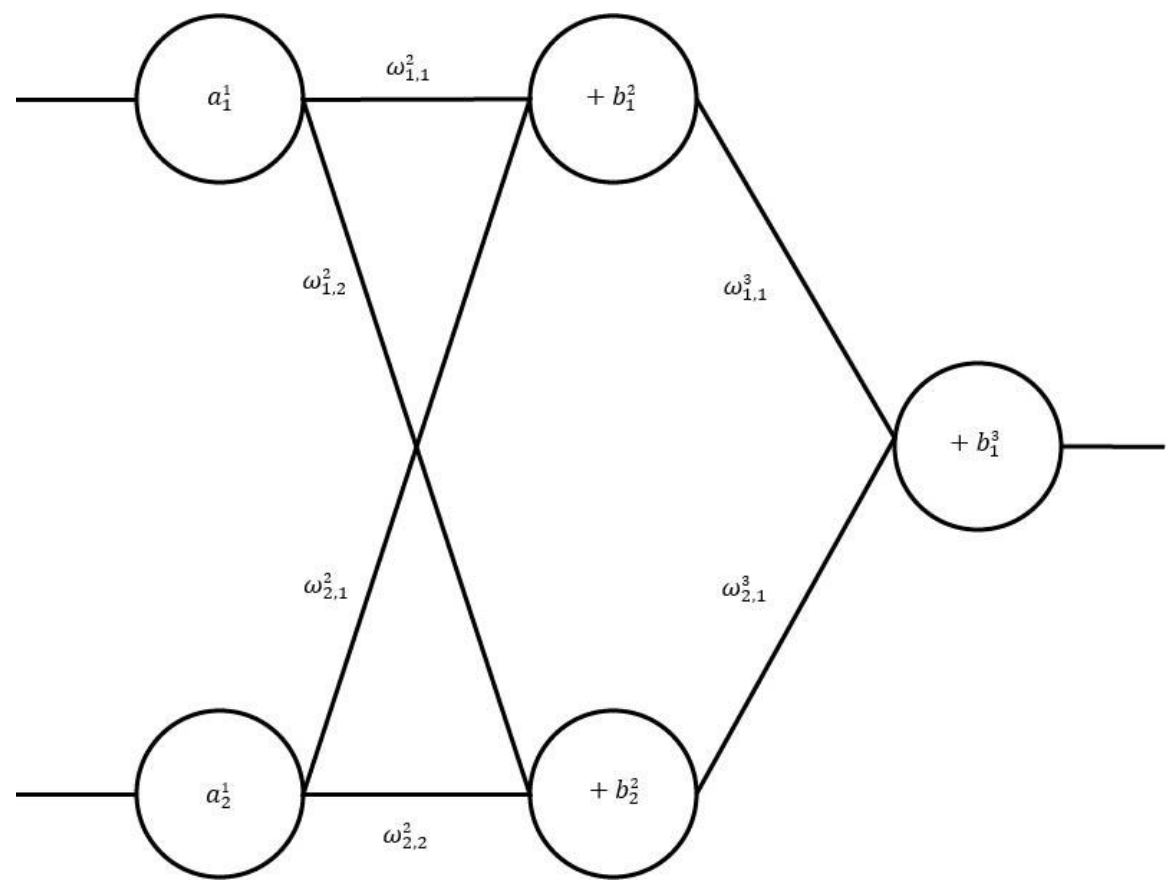

Figure 4: A three-layer network with 2 inputs, 2 nodes in the second layer, and one output.

Consider a neural network with 2 input nodes, 2 nodes in the second layer, and 1 node in the output layer. The output can be calculated as follows when the inputs are both equal to 1 , and the following weights and biases.

$$
\begin{gathered}
a_{1}^{1}=1, a_{2}^{1}=1 \\
\omega_{1,1}^{2}=0.5, \omega_{1,2}^{2}=0.5, \omega_{2,1}^{2}=0.5, \omega_{2,2}^{2}=0.5 \\
\omega_{1,1}^{3}=0.5, \omega_{2,1}^{3}=0.5 \\
b_{1}^{2}=0.5, b_{2}^{2}=0.5, b_{1}^{3}=0.5
\end{gathered}
$$

The goal is to find the activation value of the node in the last layer, but first we must find the activation values in the second layer, using (1) and (2): 


$$
\begin{aligned}
& a_{1}^{2}=\sigma\left(\sum_{i}^{2} \omega_{i, 1}^{2} a_{i}^{1}+b_{1}^{2}\right)=\sigma(0.5(1)+0.5(1)+0.5)=\sigma(1.5)=0.818 \\
& a_{2}^{2}=\sigma\left(\sum_{i}^{2} \omega_{i, 2}^{2} a_{i}^{1}+b_{2}^{2}\right)=\sigma(0.5(1)+0.5(1)+0.5)=\sigma(1.5)=0.818
\end{aligned}
$$

Then the activation value of the last node can be calculated, again using (1) and (2):

$$
a_{1}^{3}=\sigma\left(\sum_{i}^{2} \omega_{i, 1}^{3} a_{i}^{2}+b_{1}^{3}\right)=\sigma(0.5(0.818)+0.5(0.818)+0.5)=\sigma(1.32)=0.789
$$

So for the inputs of 1 , and 1 our network predicts an output value of 0.789 .

\section{GRADIENT DESCENT}

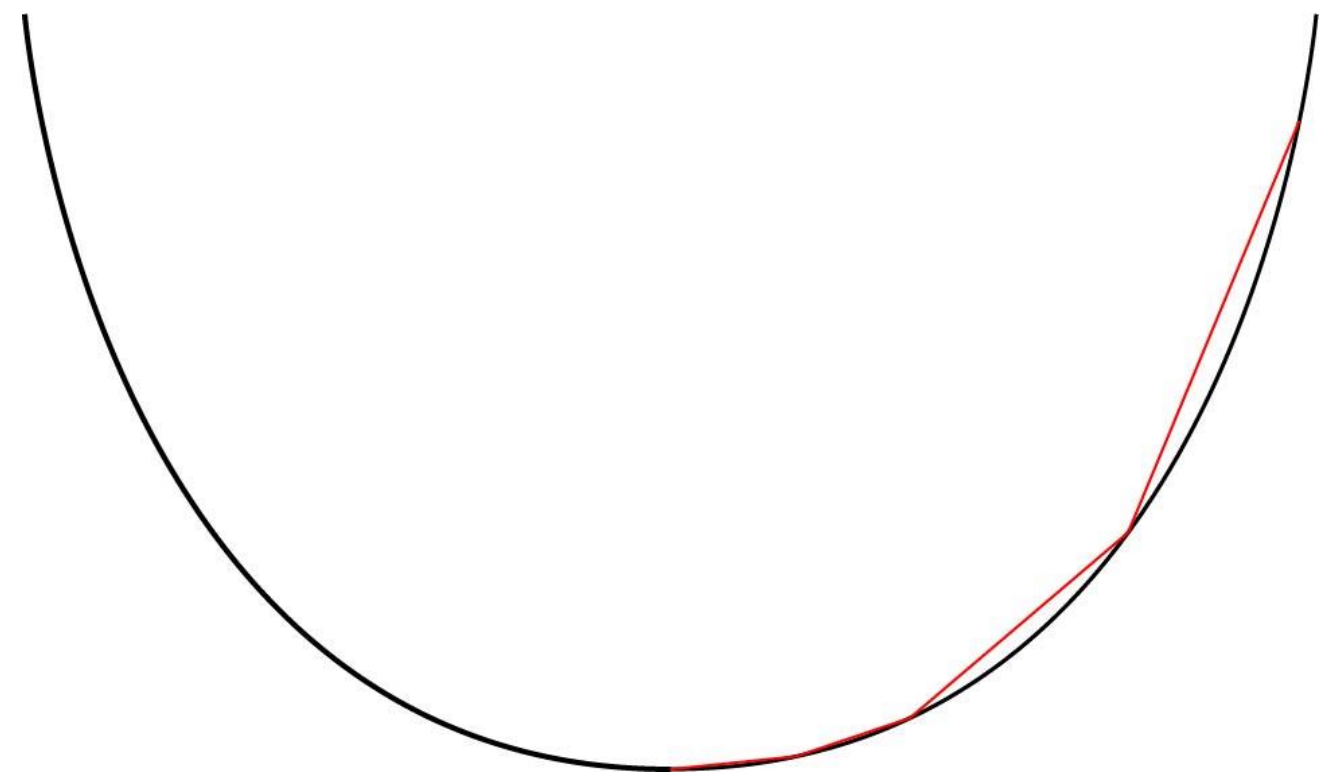

Figure 5: A sketch of a gradient descent path (red) in a parabola (black).

We will apply gradient descent to the function $f(x)=x^{2}$, starting at $\mathrm{x}=1$ using $\eta=0.4$.

We can write the update as follows:

$$
\begin{gathered}
x:=x-\eta \cdot f^{\prime}(x) \\
\text { Or } \\
x:=x-0.8 x
\end{gathered}
$$


The update can be repeated until close to a minimum value.

$$
\begin{gathered}
x:=1-0.8=0.2 \\
x:=0.2-0.8(0.2)=0.04 \\
x:=0.04-0.8(0.04)=0.008
\end{gathered}
$$

As you can see, each iteration of gradient descent is getting closer to the minimum value of the function, 0 .

\section{BACKPROPAGATION}

To update the weights of the 3-layer neural network created in Appendix I, there are a couple more pieces of information that are necessary. The first is the $\eta$ term for gradient descent, we will use $\eta=0.1$. The next is the expected output given the inputs of 1 , and 1 . We will use 1 as the expected output. Again, we have a neural network with a 2 node first layer, a 2 node second layer, and a 1 node output layer, with the following weights, biases, and inputs:

$$
\begin{gathered}
a_{1}^{1}=1, a_{2}^{1}=1 \\
\omega_{1,1}^{2}=0.5, \omega_{1,2}^{2}=0.5, \omega_{2,1}^{2}=0.5, \omega_{2,2}^{2}=0.5 \\
\omega_{1,1}^{3}=0.5, \omega_{2,1}^{3}=0.5 \\
b_{1}^{2}=0.5, b_{2}^{2}=0.5, b_{1}^{3}=0.5 \\
y_{1}=1 \\
\eta=1
\end{gathered}
$$

We start by forward propagating the network, which is already done in Appendix I. We know that:

$$
\begin{gathered}
z_{1}^{2}=z_{2}^{2}=1.5 \\
a_{1}^{2}=a_{2}^{2}=0.818 \\
z_{1}^{3}=1.32
\end{gathered}
$$




$$
a_{1}^{3}=0.789
$$

Now the delta term for the last node can be calculated, using (10):

$$
\begin{gathered}
\delta_{1}^{\mathrm{L}}=\left(a_{1}^{\mathrm{L}}-y_{1}\right) \sigma^{\prime}\left(z_{1}^{\mathrm{L}}\right)=(0.789-1) \sigma(x)(1-\sigma(x))=-0.211(0.789)(1-0.789) \\
=-0.035127069
\end{gathered}
$$

Then the delta terms for the nodes in the second layer may be calculated, using (9):

$$
\begin{aligned}
& \delta_{1}^{2}=\sum_{k}^{1} \delta_{k}^{3} \cdot \omega_{1, k}^{3} \sigma^{\prime}\left(z_{1}^{2}\right)=(-0.035127069)(0.5)(0.818)(1-0.818)=-0.00261478876 \\
& \delta_{2}^{2}=\sum_{k}^{1} \delta_{k}^{3} \cdot \omega_{2, k}^{3} \sigma^{\prime}\left(z_{2}^{2}\right)=(-0.035127069)(0.5)(0.818)(1-0.818)=-0.00261478876
\end{aligned}
$$

Now the delta values can be used to calculated the derivatives of each weight and bias, using (7) and (8):

$$
\begin{gathered}
\frac{\partial E}{\partial b_{1}^{2}}=-0.00261478876 \\
\frac{\partial E}{\partial b_{2}^{2}}=-0.00261478876 \\
\frac{\partial E}{\partial b_{1}^{3}}=-0.035127069 \\
\frac{\partial E}{\partial \omega_{1,1}^{2}}=\delta_{1}^{2} \cdot a_{1}^{1}=-0.00261478876(1)=-0.00261478876 \\
\frac{\partial E}{\partial \omega_{2,1}^{2}}=\delta_{1}^{2} \cdot a_{2}^{1}=-0.00261478876(1)=-0.00261478876 \\
\frac{\partial E}{\partial \omega_{1,2}^{2}}=\delta_{2}^{2} \cdot a_{1}^{1}=-0.00261478876(1)=-0.00261478876 \\
\frac{\partial E}{\partial \omega_{2,2}^{2}}=\delta_{2}^{2} \cdot a_{2}^{1}=-0.00261478876(1)=-0.00261478876
\end{gathered}
$$




$$
\begin{gathered}
\frac{\partial E}{\partial \omega_{1,1}^{3}}=\delta_{1}^{3} \cdot a_{1}^{2}=(-0.035127069)(0.818)=-0.02873394244 \\
\frac{\partial E}{\partial \omega_{2,1}^{3}}=\delta_{1}^{3} \cdot a_{2}^{2}=(-0.035127069)(0.818)=-0.02873394244
\end{gathered}
$$

Finally, gradient descent is used to update each weight and bias, using (4) and (5):

$$
\begin{gathered}
b_{1}^{2}:=b_{1}^{2}-\eta \cdot \frac{\partial E}{\partial b_{1}^{2}}=0.5-(1)(-0.00261478876)=0.50261478876 \\
b_{2}^{2}:=b_{2}^{2}-\eta \cdot \frac{\partial E}{\partial b_{2}^{2}}=0.5-(1)(-0.00261478876)=0.50261478876 \\
b_{1}^{3}:=b_{1}^{3}-\eta \cdot \frac{\partial E}{\partial b_{1}^{3}}=0.5-(1)(-0.035127069)=0.535127069 \\
\omega_{1,1}^{2}:=\omega_{1,1}^{2}-\eta \cdot \frac{\partial E}{\partial \omega_{1,1}^{2}}=0.5-(1)(-0.00261478876)=0.50261478876 \\
\omega_{2,1}^{2}:=\omega_{2,1}^{2}-\eta \cdot \frac{\partial E}{\partial \omega_{2,1}^{2}}=0.5-(1)(-0.00261478876)=0.50261478876 \\
\omega_{1,2}^{2}:=\omega_{1,2}^{2}-\eta \cdot \frac{\partial E}{\partial \omega_{1,2}^{2}}=0.5-(1)(-0.00261478876)=0.50261478876 \\
\omega_{2,1}^{3}:=\omega_{2,1}^{3}-\eta \cdot \frac{\partial E}{\partial \omega_{2,1}^{3}}=0.5-(1)(-0.02873394244)=0.52873394244 \\
\omega_{1,1}^{3}:=\omega_{1,1}^{3}-\eta \cdot \frac{\partial E}{\partial \omega_{1,1}^{3}}=0.5-(1)(-0.02873394244)=0.52873394244 \\
:=\omega_{2,2}^{2}-\eta \cdot \frac{\partial E}{\partial \omega_{2,2}^{2}}=0.5-(1)(-0.00261478876)=0.50261478876 \\
\omega_{2,2}
\end{gathered}
$$

\section{SOURCE CodE}

The following is the raw C\# code that I wrote to calculate the weights and biases, as well as create the model that was arrived at as a solution to the problem. 


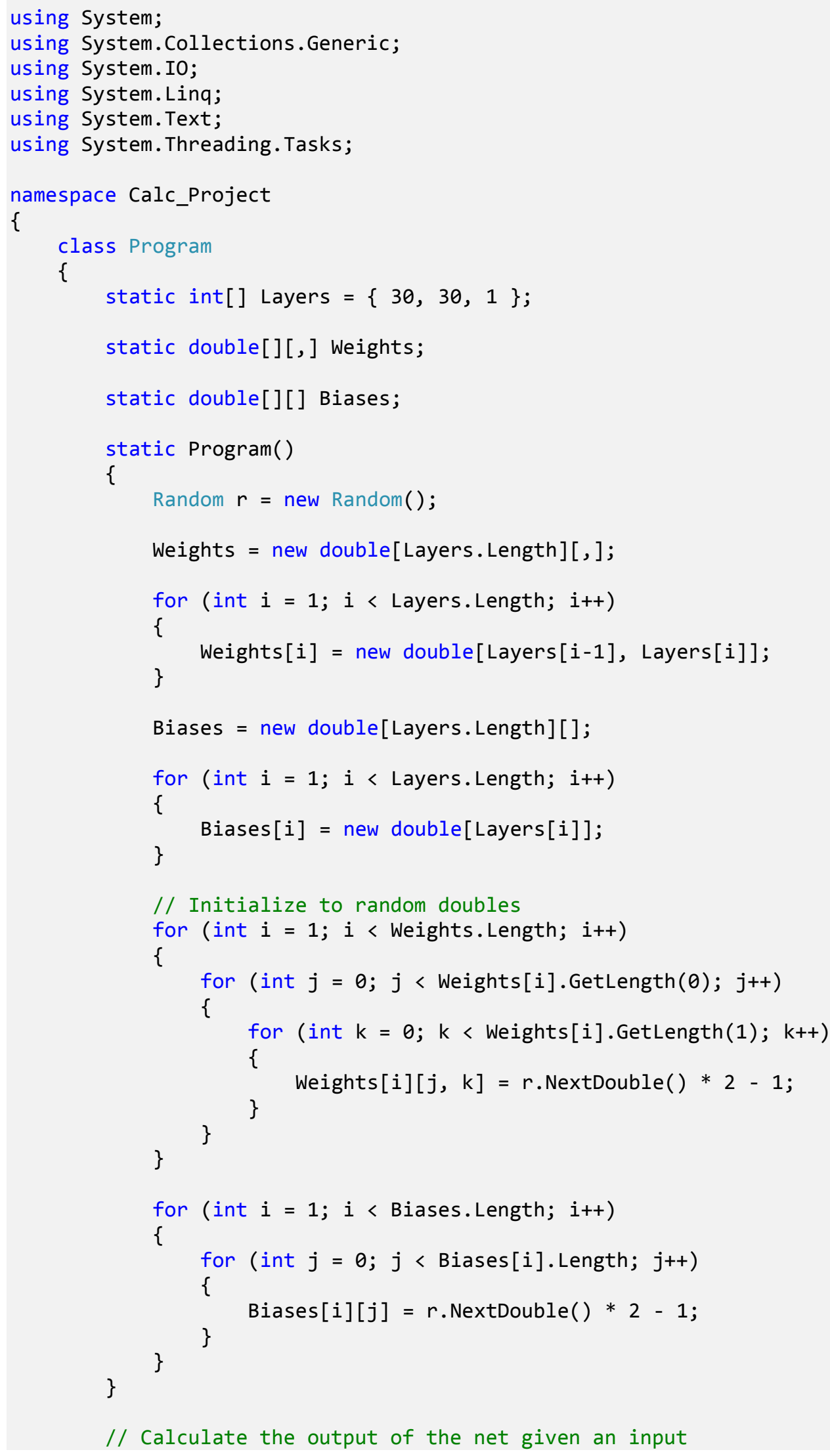




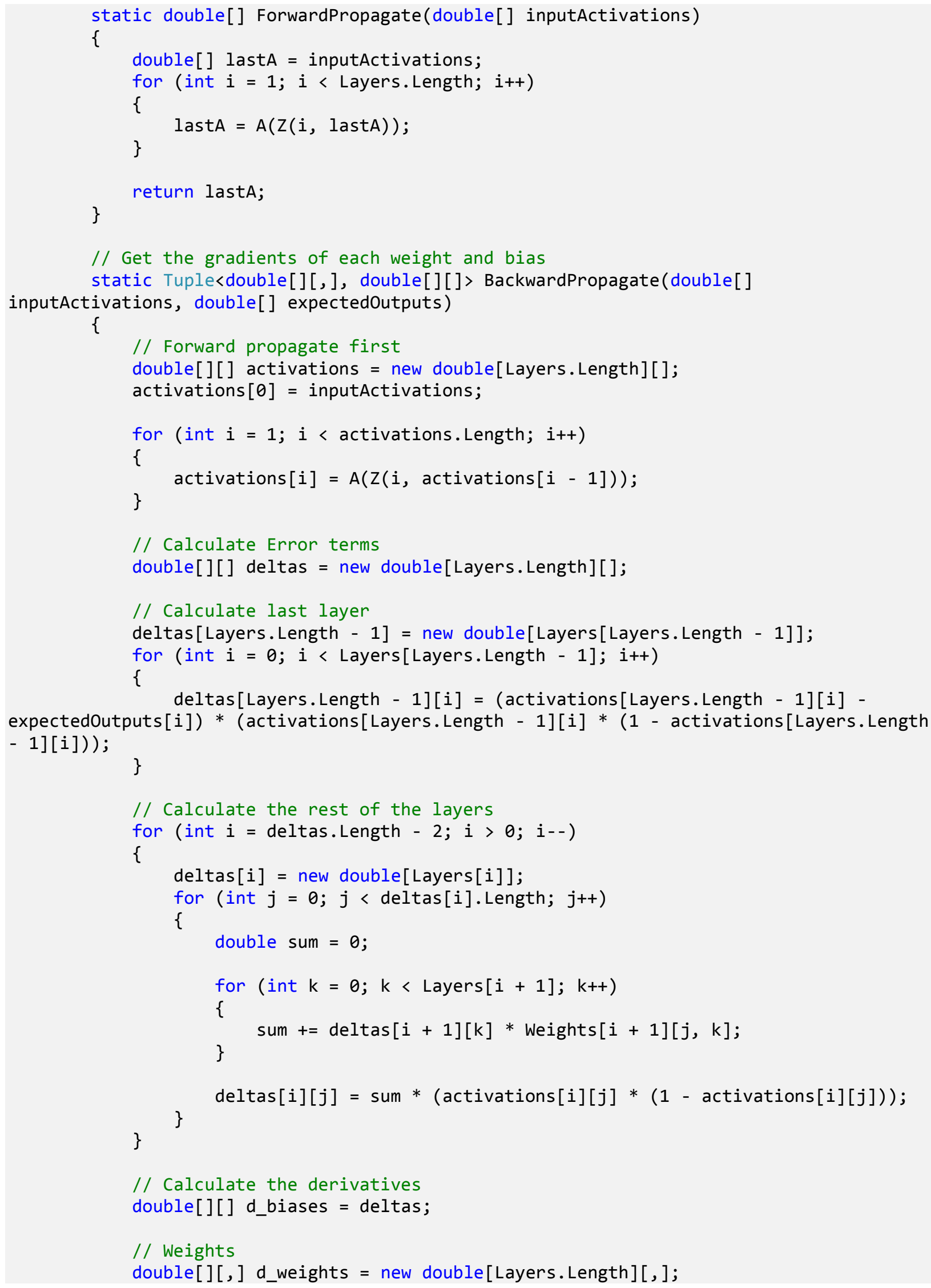


for (int $1=1 ; 1<d$ weights. Length; $1++$ )

\{

d_weights $[1]=$ new double $[\operatorname{Layers}[1-1]$, Layers $[1]]$;

for (int $i=0$; $i<d$ weights[1].GetLength(0); $i++$ )

\{

for (int $j=0 ; j<d \_w e i g h t s[1]$.GetLength(1); j++)

\{ \} d_weights $[1][i, j]=\operatorname{deltas}[1][j] *$ activations $[1-1][i]$;

\}

\}

\}

return Tuple.Create(d_weights, d_biases);

// Estimate the values of the derivatives, to check the math done in the other BackPropagate function

static Tuple<double[][,], double[][]> BackwardPropagateEstimate(double[] inputActivations, double[] expectedOutputs, double epsilon)

\{

double[][] d_biases = new double[Layers.Length][];

double []$[$,$] d_weights = new double[Layers.Length][,];$

// Estimate bias derivatives

for (int $1=1$; $1<d \_b i a s e s . L e n g t h ; 1++$ )

\{

d_biases[1] = new double[Layers[1]];

for (int $j=0$; $j<d$ biases[1]. Length; $j++$ )

\{

// For each bias estimate the gradient when changed slightly

Biases[1][j] += epsilon;

expectedOutputs);

double loss1 = Loss(ForwardPropagate(inputActivations),

Biases[1][j] -= 2*epsilon;

double loss2 = Loss(ForwardPropagate(inputActivations),

expectedOutputs);

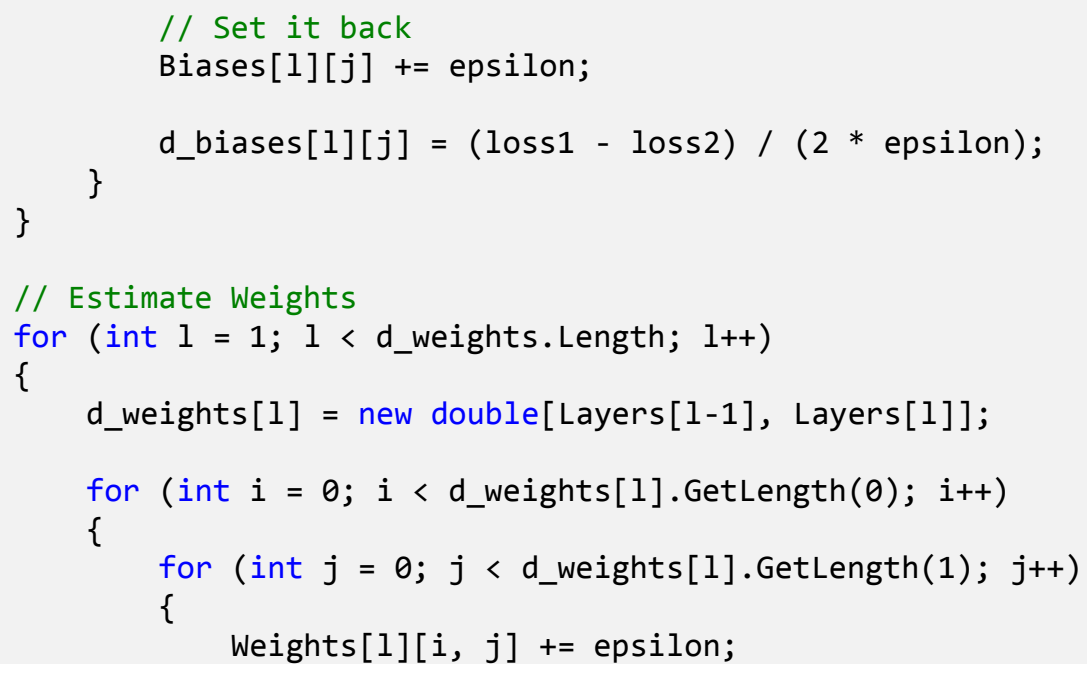




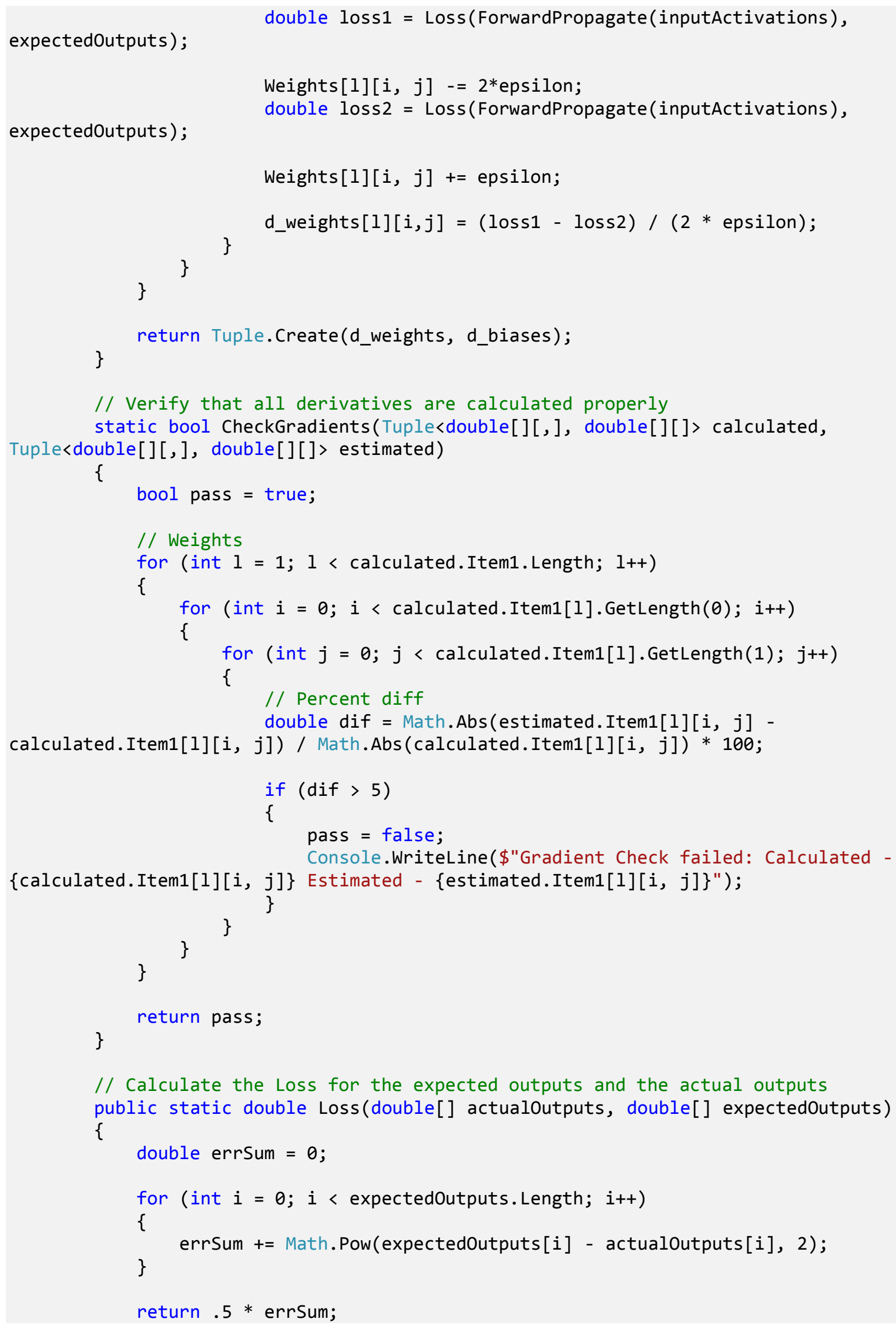




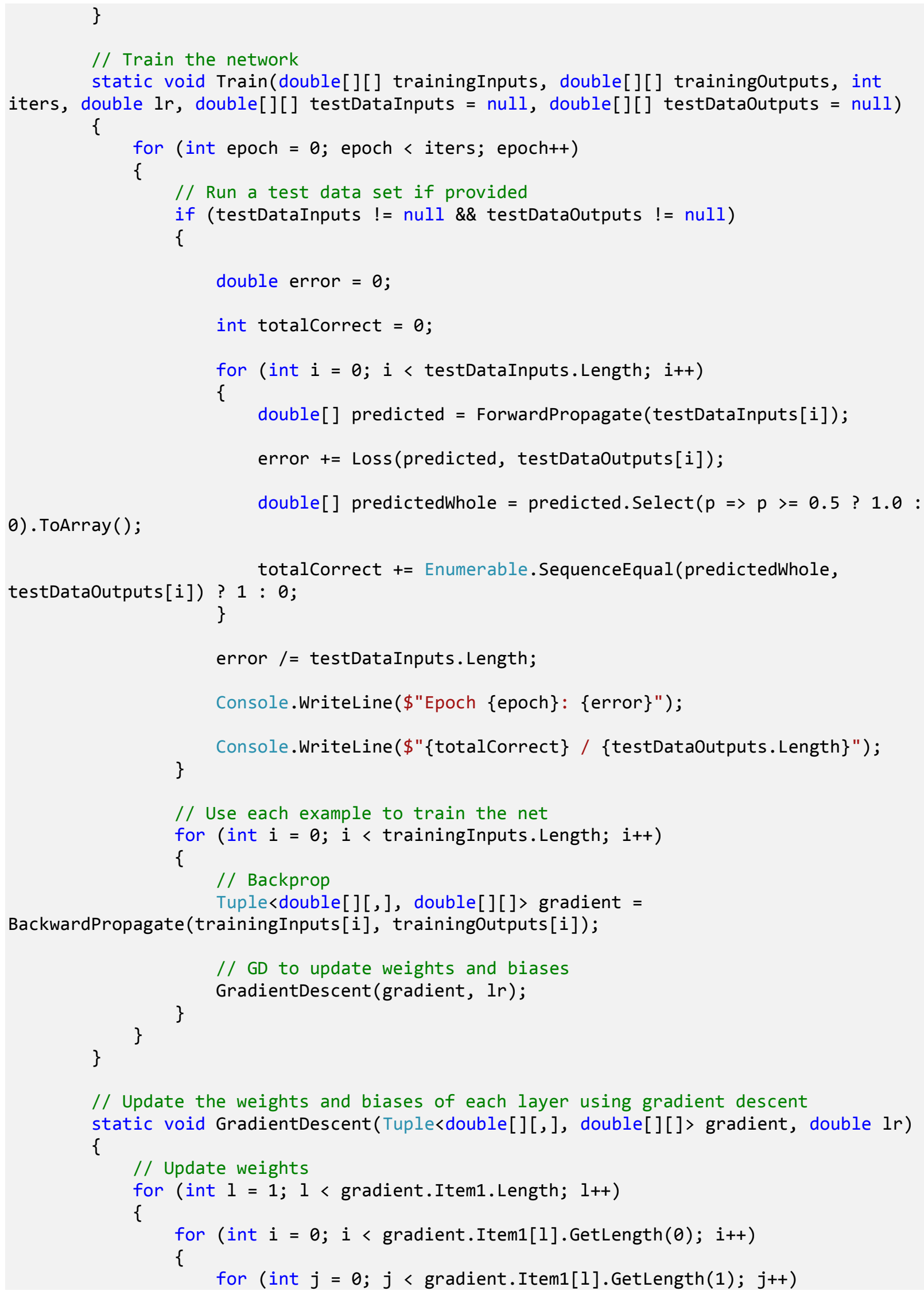




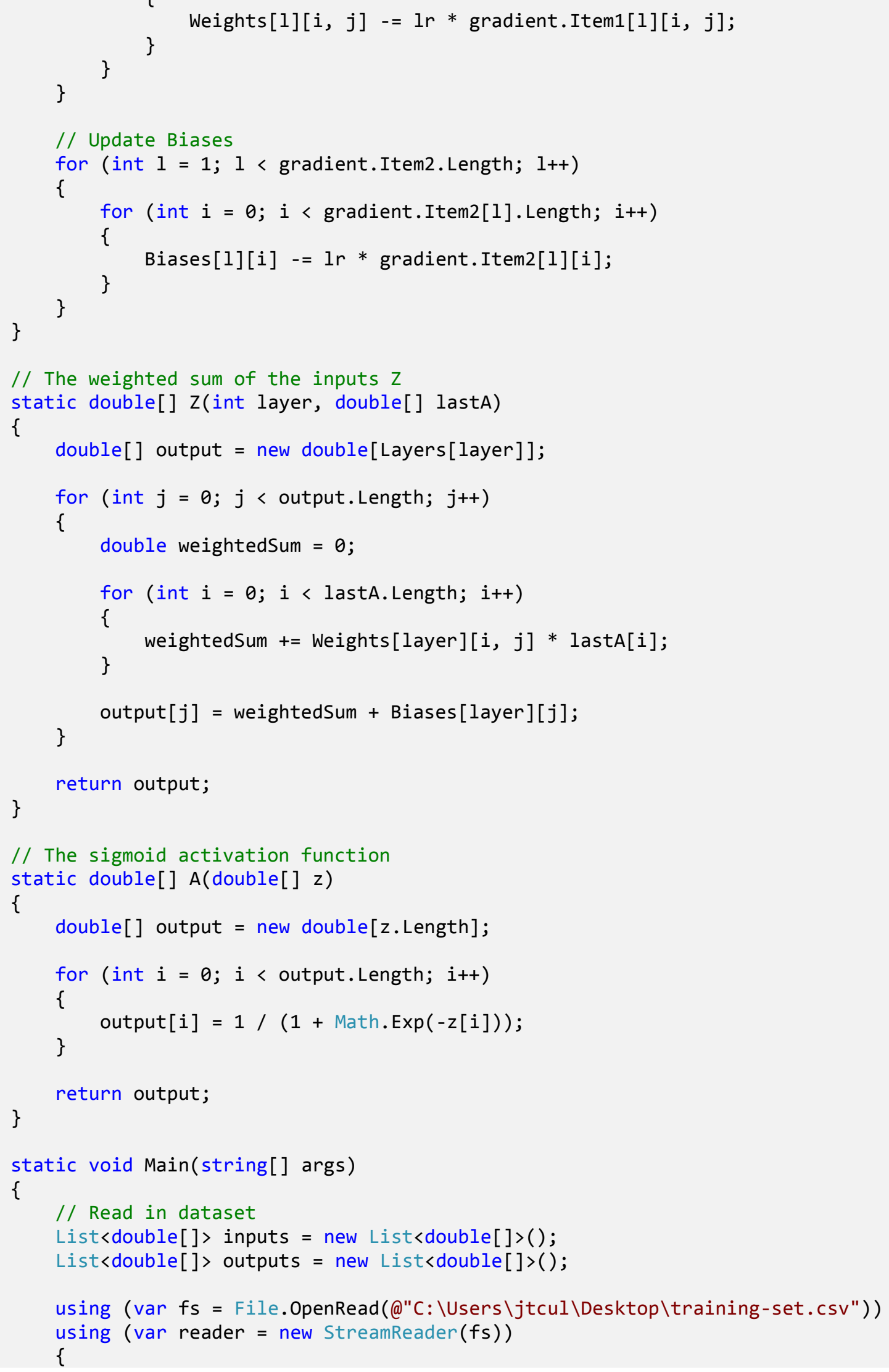




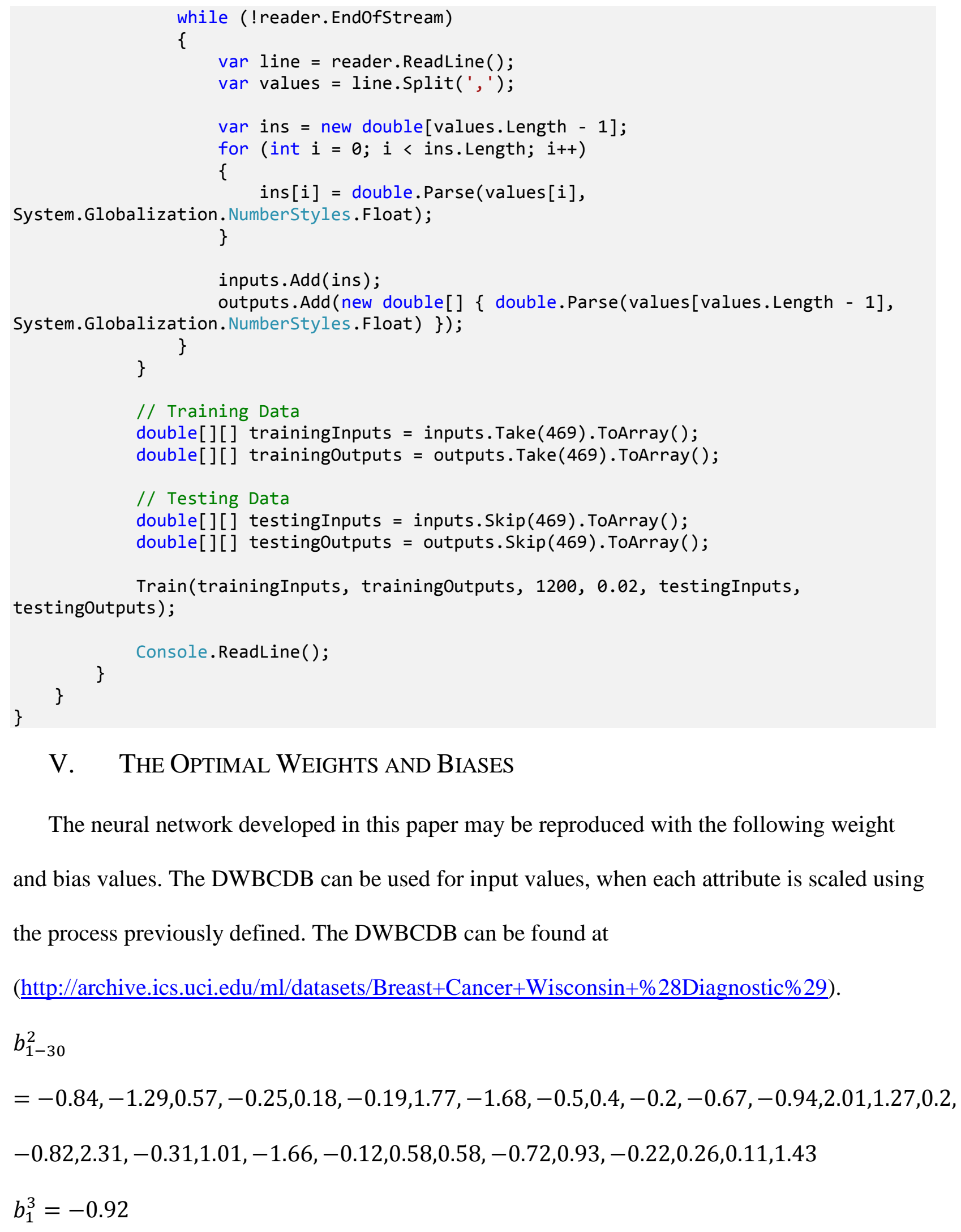

\section{ThE OPtimal WeIGHTS AND BIASES}

The neural network developed in this paper may be reproduced with the following weight and bias values. The DWBCDB can be used for input values, when each attribute is scaled using the process previously defined. The DWBCDB can be found at (http://archive.ics.uci.edu/ml/datasets/Breast+Cancer+Wisconsin+\%28Diagnostic\%29).

$$
\begin{aligned}
& b_{1-30}^{2} \\
& =-0.84,-1.29,0.57,-0.25,0.18,-0.19,1.77,-1.68,-0.5,0.4,-0.2,-0.67,-0.94,2.01,1.27,0.2, \\
& -0.82,2.31,-0.31,1.01,-1.66,-0.12,0.58,0.58,-0.72,0.93,-0.22,0.26,0.11,1.43 \\
& b_{1}^{3}=-0.92
\end{aligned}
$$




$$
\begin{aligned}
& \omega_{1,1-30}^{2} \\
& =-1.02,-0.69,-0.79,0.56,-0.13,0.52,0.09,0.27,0.1,-0.6,-0.49,0.25,0.26,0.48,-0.07,0.76,-0.54,0.08 \text {, } \\
& 0.89,-0.01,-0.36,0.61,0.48,0.44,-1.11,0.57,0.12,0.97,0.61,-0.95 \\
& \omega_{2,1-30}^{2} \\
& =0.86,0.1,0.26,-0.67,0.98,1.06,-1.21,-0.27,-0.42,0.55,0.74,-0.26,0.01,-1.09,-0.31,0.07 \text {, } \\
& -0.71,0.04,0.08,-0.35,0.17,0.74,-0.05,0.56,0.44,-0.54,-0.25,-0.1,0.24,0.14 \\
& \omega_{3,1-30}^{2} \\
& =-0.78,0.28,0.16,0.32,-0.58,0.37,0.41,0.29,0.39,0.05,0.82,1.12,-0.44,-0.66,0.5,0.89,-0.25,0.32 \text {, } \\
& -0.27,-0.1,0.51,-0.91,-0.16,-0.15,-0.17,-0.79,-0.89,0.8,-0.38,0.43 \\
& \omega_{4,1-30}^{2} \\
& =0.13,0.33,-0.01,0.67,0.23,-0.63,-0.15,1.15,0.67,0.52,0.64,0.46,0.66,-1.09,-0.16,0.07,0.35,-0.77 \text {, } \\
& -0.93,0.38,1.1,0.4,-0.94,-0.76,0.65,-0.82,-0.59,-0.52,-0.93,-0.96 \\
& \omega_{5,1-30}^{2} \\
& =0.1,0.53,-0.69,-0.82,0.73,-0.48,-0.28,0.11,0.12,0.19,-0.15,0.04,0.68,0.27,0.63,-0.91,-0.42 \text {, } \\
& -0.03,0.81,0.91,0.03,0.53,0.7,-0.04,0.54,0.75,0,-0.02,0.53,-0.28 \\
& \omega_{6,1-30}^{2} \\
& =0.85,-0.78,0.79,0.52,-0.67,0.85,0.14,0.2,-0.81,-0.43,-0.6,-0.86,0.2,0.26,0.31,-0.09,0.54,0.5 \text {, } \\
& -0.26,0.95,0.48,0.67,-0.15,-0.93,0.17,0.87,-0.91,0.37,-0.43,1.04 \\
& \omega_{7,1-30}^{2} \\
& =0.44,1.09,-0.02,-0.34,0.3,-0.91,-0.55,-0.15,0.27,0.88,0.45,0.49,-0.32,-0.49,-0.11,-0.3,-0.58 \text {, } \\
& -1.1,-0.96,-1.05,-0.14,-0.79,-1.15,0.91,-0.1,-1.15,-0.95,0.64,-0.71,-0.95 \\
& \omega_{8,1-30}^{2} \\
& =0.57,0.81,-0.5,-0.02,-0.01,-0.18,-1.01,0.95,0.8,-0.09,0.86,0.88,1.19,-0.85,-1.12,-0.5,0.68 \text {, }
\end{aligned}
$$




$$
\begin{aligned}
& -0.64,-0.41,-1.41,1.32,0.32,-0.07,0.78,0.29,0.08,0.38,0.74,-0.06,-0.45 \\
& \omega_{9,1-30}^{2} \\
& =-1,0.29,0.81,-0.52,-0.91,0.88,-0.76,0.73,-0.73,-0.31,-0.91,-0.13,0.13,0.41,0.34,-0.28,-0.73 \text {, } \\
& 0.76,0.3,-0.27,-0.21,-0.24,0.03,0.99,-0.02,-0.51,-0.94,0.34,-0.17,0.46 \\
& \omega_{10,1-30}^{2} \\
& =0.42,-0.3,-0.66,-0.56,-0.29,-1.03,0.87,0.17,-0.16,-0.45,-0.48,0.46,-0.16,1.1,-0.4,-0.26,0.94 \text {, } \\
& -0.4,-0.38,0.12,-0.02,-0.92,-0.54,-0.62,-0.68,0.32,-0.25,0.82,0.4,0.55 \\
& \omega_{11,1-30}^{2} \\
& =0.77,0.24,-0.42,0.11,0.26,0,-0.98,0.36,-0.21,0.08,0.24,0.14,-0.18,-0.99,-1.52,0.29,1.04,-0.88 \text {, } \\
& 0.64,-1.31,0.04,1.06,-0.69,0.96,-0.88,-0.01,-0.32,0.92,-0.84,-0.06 \\
& \omega_{12,1-30}^{2} \\
& =0.28,0.55,1.05,0.19,-0.22,0.84,-0.33,-0.88,-0.52,0.21,-0.92,0.2,0.43,0.79,1.12,-0.64,0.52,0.98 \text {, } \\
& -0.71,0.03,0.37,0.71,-0.78,0.11,0.11,0.9,-0.96,0.04,-0.55,0.96 \\
& \omega_{13,1-30}^{2} \\
& =-0.7,0.01,-0.74,0.31,0.74,0.25,-0.47,0.52,1,-0.68,-0.04,0.7,-0.46,-0.88,-1.09,0.25 \text {, } \\
& -0.38,-0.29,-1.01,-1.25,0.99,0.12,0.38,0.45,0.7,0.41,0.64,-0.14,-0.91,-0.29 \\
& \omega_{14,1-30}^{2} \\
& =0.73,0.74,0.5,-0.09,-0.12,0.28,-0.71,-0.21,1.01,-0.32,0.63,-0.41,0.93,-1.46,0.57,-1.01 \text {, } \\
& 0.55,0.1,-0.03,0.43,0.75,-0.01,-0.16,-0.53,-0.99,0.03,0.22,0.1,0.21,-1.12 \\
& \omega_{15,1-30}^{2} \\
& =0.55,1.2,-0.21,0.06,-0.57,0.39,0.22,1.18,0.55,-0.17,-0.29,-0.62,0.59,-0.05,-0.9,0.31,0.5 \text {, } \\
& -0.05,0.42,0.28,-0.56,0.99,0.43,-0.66,0.32,-0.52,-0.69,0.34,-0.61,0.15
\end{aligned}
$$




$$
\begin{aligned}
& \omega_{16,1-30}^{2} \\
& =-0.93,0.05,0.32,0.41,0.08,0.64,0.72,-0.72,0.06,-0.49,-0.8,0.52,-1.09,-0.14,0.91,0.3,0.42,1.63 \text {, } \\
& 0.96,0.29,0.23,0.64,0.89,0.38,1.03,0.98,-0.82,-0.34,0.63,1.04 \\
& \omega_{17,1-30}^{2} \\
& =0.16,-0.65,0.15,0.88,-0.22,-0.09,-0.05,0.6,-0.78,-0.04,-0.87,-0.25,-0.91,0,0.55,-0.48 \text {, } \\
& 0.77,0.76,0.02,-0.73,0.05,-0.42,0.41,0.7,0.75,-0.63,-0.93,0.57,0.71,0.36 \\
& \omega_{18,1-30}^{2} \\
& =0.37,-0.78,0.2,0.75,0.35,0.95,-0.46,-0.68,0.11,-0.64,-0.72,-0.8,0.43,0.17,-0.1,0.13,0.56 \text {, } \\
& -0.12,0.98,0.47,0.71,-0.58,0.76,0.14,-0.57,-0.38,-0.02,0.38,-0.82,-0.64 \\
& \omega_{19,1-30}^{2} \\
& =-0.15,-0.02,0.9,-0.19,0.56,0.64,0.69,-0.91,0.47,0.56,-1.07,0.39,-0.7,-0.01,-0.62,0.04 \text {, } \\
& 0.06,-0.13,0,-0.19,-0.48,0.4,0.69,-0.74,0.52,0.1,-0.31,-0.94,0.3,-0.2 \\
& \omega_{20,1-30}^{2} \\
& =-0.04,-0.23,-0.75,-0.21,0.49,-0.12,-0.59,-0.67,0.23,0.05,0.67,0.3,-0.04,-0.31,0.67 \text {, } \\
& -0.6,-1.09,0.51,0.15,0.72,0.17,-0.52,-0.42,0.46,0.69,0.55,0.8,0.52,0.47,-0.33 \\
& \omega_{21,1-30}^{2} \\
& =-0.13,0.87,-0.47,0.29,-0.38,0.01,-0.45,1.26,0.36,0.27,0,0.07,0.4,0.14,-0.69,0.28,-0.28 \text {, } \\
& -0.76,-0.18,-0.58,0.66,0.66,-0.44,0.82,-0.09,-0.88,-0.46,-0.27,0.15,-1.44 \\
& \omega_{22,1-30}^{2} \\
& =-0.02,0.25,-0.44,0.35,-0.97,-0.7,-1.71,0.11,1.05,-0.54,0.32,0.09,1.37,-0.19,-0.98 \text {, } \\
& -0.31,0.9,-1.54,-0.49,-1.53,0.96,-0.33,-0.73,-0.58,0.26,-0.05,-0.65,0.39,0.34,-1.52 \\
& \omega_{23,1-30}^{2} \\
& =-0.67,-0.01,-1.15,0.47,-0.9,-0.86,-0.02,-0.24,0.9,-0.32,0.25,-0.53,0.71,-1.05 \text {, }
\end{aligned}
$$




$$
\begin{aligned}
& -1.3,-0.71,0.87,-0.68,-0.08,-1,0.48,-0.9,-1.16,0.07,-0.35,-0.66,0.93,-0.16,0.19,-0.65 \\
& \omega_{24,1-30}^{2} \\
& =0.84,0.52,-0.74,0.94,0.97,-0.65,-1.31,0.45,1.08,-0.21,0.48,0.62,0.03,-1.68,-0.39,-0.13 \text {, } \\
& 0.38,-1.65,-0.81,-0.39,1.42,0.56,-0.99,0.17,-0.11,-0.89,-0.39,0.26,-0.93,0.15 \\
& \omega_{25,1-30}^{2} \\
& =-0.96,-0.17,-0.31,0.5,-0.37,-0.99,0.06,1.3,-0.35,0.74,-0.82,0.13,0.19,-1.34,-0.1,0.63 \text {, } \\
& 0.55,-1.29,0.79,-1.46,0.92,0.78,-0.66,1.08,-0.38,-0.87,-0.3,0.63,-0.74,0.05 \\
& \omega_{26,1-30}^{2} \\
& =0.67,-0.21,-0.26,-0.65,-0.37,-0.32,-0.51,0.26,0.2,0.21,0.45,0.13,0.54,-0.25,0.58,-0.01 \text {, } \\
& -0.67,-0.34,0.93,0.64,-0.08,-0.43,0.57,-0.88,-0.9,-0.36,-0.82,-0.1,-0.51,-0.63 \\
& \omega_{27,1-30}^{2} \\
& =0.25,1.13,0.23,0.25,-0.98,-0.15,-1.36,0.11,0.85,-0.3,0.53,1.07,-0.14,-0.9,-0.84,-0.79 \text {, } \\
& 1.2,-1.42,-0.24,0.35,-0.52,-0.3,0.47,-0.01,-0.72,-0.07,0.82,-0.49,-0.81,-1.22 \\
& \omega_{28,1-30}^{2} \\
& =-0.72,0.43,-0.18,0.56,0.88,0.72,0.17,0.34,-0.43,-0.49,-0.06,1.02,-0.48,-0.23,-1.15 \text {, } \\
& 0.19,1.1,-0.74,0.64,0.29,0.02,-0.82,-1.02,-0.43,-1.17,-0.47,-0.78,-0.36,-0.55,-0.48 \\
& \omega_{29,1-30}^{2} \\
& =-0.04,0.83,-0.8,0.8,-0.81,-1.03,-0.77,0.92,0.08,-0.1,-0.28,0.26,0.75,-1.12,-0.25 \text {, } \\
& -0.7,-0.53,-1.05,-0.21,-0.24,0.73,0.26,-0.42,-0.46,0.47,-0.89,0.17,-0.21,-0.16,-0.28 \\
& \omega_{30,1-30}^{2} \\
& =0.38,-0.01,-0.6,0.71,0.36,0.09,0.02,-0.14,0.19,-0.95,-0.29,-0.64,0.8,-0.41,0.14,0.41 \text {, } \\
& -0.41,0.81,0.46,0.68,-0.37,0.89,0.77,0.27,0.6,0.77,-0.08,-0.92,0.89,0.71
\end{aligned}
$$




$$
\begin{aligned}
& \omega_{1-30,1}^{3} \\
& =0.02,2.9,-1.88,1.57,-0.38,-0.89,-3.35,2.86,2.03,-0.03,0.97,1.31,2.12,-3.58,-3.33,-0.36,1.68, \\
& -4.54,-0.28,-3.12,2.89,0.52,-2.06,0.88,-0.83,-2.08,-0.35,0.94,-1.03,-2.66
\end{aligned}
$$

